\title{
The efficacy of intra-articular PRP, Ozone and Ozone+PRP injections in patients with osteoarthritis.
}

\author{
Lale Yeprem ${ }^{1}$, Aysegul Ellialtioglu22, Jose Baeza-Noci³ \\ ${ }^{1}$ Private Ozone Therapy Clinic, Istanbul, Turkey. 2Private TEM Hospital, Istanbul, Turkey. ${ }^{3}$ School of Medicine, Valencia \\ University, Spain.
}

\section{子े OPEN ACCESS}

\section{Citation \\ Yeprem L, Ellialtioglu A, Baeza-Noci J. The efficacy of intra-articular PRP, Ozone and Ozone+PRP injections in patients with osteoarthritis.. J Ozone Ther. 2018:2(3) \\ doi: 10.7203/jo3t.2.3.2018.10904}

\section{Academic Editor \\ Jose Baeza-Noci, \\ School of Medicine, Valencia \\ University, SPAIN}

\section{Editor}

World Federation of Ozone Therapy, Bologna, ITALY

\section{Received}

October 10, 2017

\section{Accepted}

January 21, 2018

\section{Published}

December 15, 2018

\section{Intellectual Property}

Yeprem L.

This is an open access article distributed under the terms of the Creative Commons Attribution License (CC BY 4.0), which permits unrestricted use, distribution, and reproduction in any medium, provided the original author and source are credited.

\section{Author Information} lale@ozon.com.tr

\section{ABSTRACT}

Osteoarthritis $(\mathrm{OA})$ is the most common form of arthritis and some joints are effected more than others. The prevalence of OA increases with age and it represents the major problem for functional impairment in older patients. Non-surgical treatment with oral drugs is not suitable for many patients suffering from gastric disorders, high blood pressure or different heart diseases. Intra-articular injections are a promising solution in these cases.

This study was performed to compare the efficacy and safety of ozone injections, PRP (platelet rich plasma) injections and combined ozone plus PRP injections in patients with symptomatic knee OA.

120 patients were diagnosed of OA according to the criteria of the American College of Rheumatology and included into this study. Patients were randomly divided into three equal groups (40 patients each group). We include patients whose VAS score was 5 or above. Patients with inflammatory, endocrine and metabolic disturbances, and patients who had meniscectomy within the past 10 years, extra articular surgery within the last year, arthrocentesis in last 6 months or any drug given intra-articular were not included. We did not include either patients with misalignment.

Patients in the first group were treated with intra-articular injections of ozone/ oxygen gas mixture 2 times/week, at $10 \mu \mathrm{g} / \mathrm{mL}$ concentration and a volume of $5 \mathrm{~mL}$ for a total of 12 injections.

Patients in the second group were treated only with PRP injections once a week for a total of 3 times.

Patients in the third group were treated with intra-articular injections of Ozone+PRP $(10 \mu \mathrm{g} / \mathrm{ml}$ concentration, $5 \mathrm{ml}$ volume $)$ once a week for a total of 3 times.

The pain levels of patients were measured with Visual Analog Scale (VAS). Side effects were collected using an open list record.

All the groups improved their baseline VAS in a significant way, although the group with the best results was group 3 with the combination of ozone and PRP in the same injection.

We are planning further studies including systemic ozone in order to improve more the pain in our patients.

Keywords: Ozone knee injection; PRP knee injection; Osteoarthritis. 


\section{Introduction}

Osteoarthritis (OA) is a degenerative disease associated with the deterioration of the processes of production and destruction of cartilage and synovial tissues caused by various traumatic, biomechanical, inflammatory or genetic factors.

This study aims to evaluate the response of $O A$ by intra-articular injection of Ozone, Ozone+PRP and PRP alone in patients with knee $\mathrm{OA}$.

Even though OA can be seen in various joints, particularly knee and hip joints are more common, when the spine is affected, the results can be severe.

Knee OA is often bilateral, more frequent in women and symptomatic knee OA prevalence in our country (Turkey) is reported to be $14.8 \%$ [1].

When the results from various studies were analyzed, it was found that among causes of disability $O A$ is in 7th place among women, 12th place among men and 5th place among the elderly population [2].

In the United States (USA), the percentage of symptomatic OA above 30 years old is about $6 \%$ in hips and $3 \%$ in knees [3].

There are several alternative treatment options for pain relief, oral or topical medications, injections, physical therapy and rehabilitation treatments apart from surgical approaches [4].

In this study, we investigated how single and combined treatment of intra-articular injections of ozone, PRP or both can be an alternative treatment option in knee OA.

Ozone can be used in inflammatory and degenerative diseases of the musculoskeletal system, showing anti-inflammatory and anti-oxidative effects. Clinical studies evaluating the effects of ozone on the musculoskeletal system are being published $[5,6]$.

PRP (platelet rich plasma) is the component that is obtained by centrifugation of whole blood and it contains 1.1-1.4 million platelets $/ \mathrm{mL}$ depending on the patient's platelet level in the whole blood (5 times the normal amount). The presence of numerous growth factors in its content has led to the use of PRP injections in the treatment of various musculoskeletal disorders [7].

PRP is also a known method of tissue healing. In our study we have investigated, whether the combined use of Ozone+PRP has higher effect than ozone or PRP alone $[8,9]$.

\section{Patients and methods}

225 attended patients with knee osteoarthritis between 2017 January to 2018 December were evaluated clinically with Visual Analog Scale (VAS) before the treatments. Knee X-ray and MRI views were evaluated by Radiologist and Physical Rehabilitation doctors. We included patients grade 2 and 3 OA according to Kellgren classification but it was not a selection criteria. 
Inclusion criteria: Out of these 225 patients, 120 whose VAS was higher than 5 were included in the study, without age, gender or X-ray Kellgren score discrimination.

Exclusion criteria: Patients with inflammatory, endocrine and metabolic disturbances, and patients who had meniscectomy within the past 10 years, extra articular surgery within the last year, arthrocentesis in last 6 months or any drug given intra-articular were not included. We did not include patients with misalignment either.

We treated 185 knees from this group of 120 patients. 44 males and 76 females of 120 patients were found to have an average age of 56.7.

Patients were randomly divided in to 3 treatment groups, using a simple randomization computer program (SPSS 17.0).

1. Intra-articular Ozone injections

2. Intra-articular PRP injections

3. Intra-articular Ozone+PRP injections

In the First group: $5 \mathrm{~mL}$ of ozone/oxygen gas mixture at $10 \mu \mathrm{g} / \mathrm{mL}$ concentration was injected into the knee of the patients 2 times a week for 12 sessions. Ozone/oxygen gas mixture was taken from TURKOZONE BLUE Medical Ozone Generator (Ozon Saglik Hiz.Ltd Sti, İstanbul-Turkey) (Image 1). We used siliconized ozone resistant Omnifix $10 \mathrm{ml}$ syringes (BRAUN, Melsungen, Germany) with and Millex millipore bacterial filters (Merck Millipore Ltd. Ireland, Tullagreen. Carrigtwohill, County Cork, Ireland) and 27G, $40 \mathrm{~mm}$ Ayset needle (AYSET Tıbbi Ürünler, Adana, Turkey). Through a lateral approach to the knee, we injected $5 \mathrm{~mL}$ of ozone/oxygen gas mixture into the knee joint.

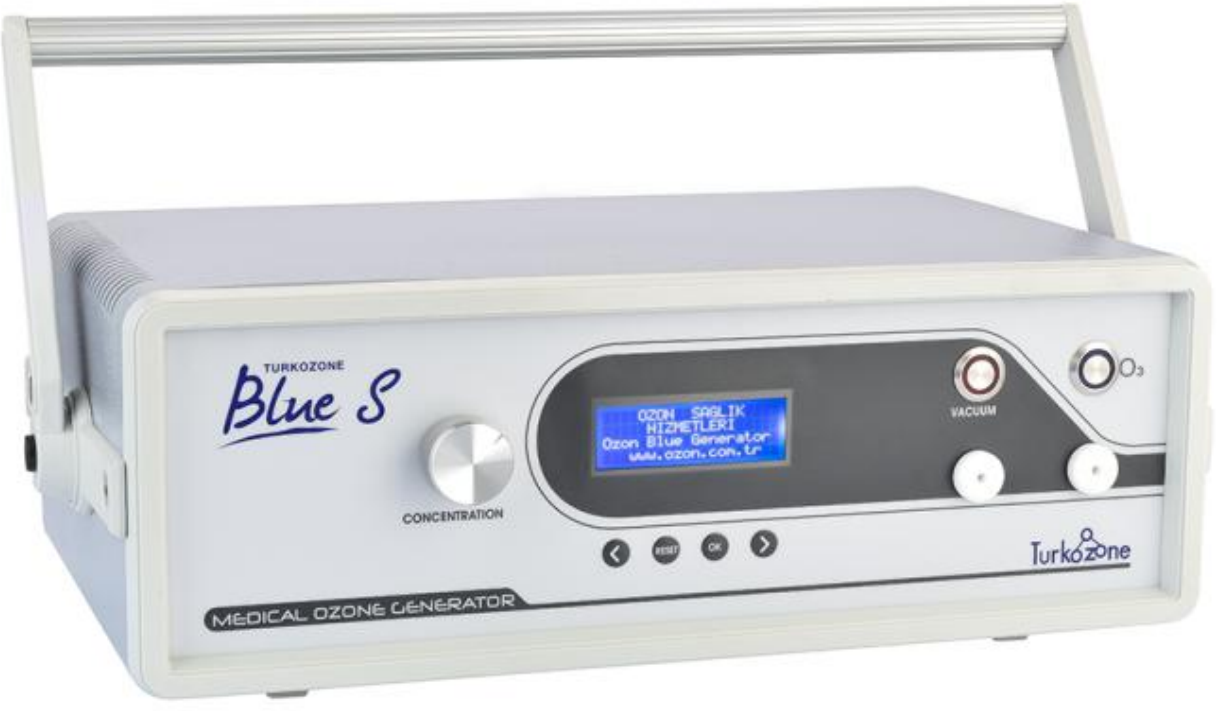

Image 1. Turkozone Blue Medical Ozone Generator (Ozon Saglik Hiz.Ltd Sti, İstanbulTurkey) 
In the Second group: PRP was injected into the knee of the patient 3 times (once a week).

Electromag Centrifuge 415P (Manufacturer Electro-Mag Medikal İstanbul/Turkey) has been used for preparing the PRP.

$10 \mathrm{~mL}$ of blood were taken from the patient's vein and mixed with 1 $\mathrm{mL}$ of sodium citrate $3.13 \%$ using Easy PRP kit (NEOTEC Bioteknoloji, İstanbul, Turkey) (Image 2), and the first centrifugation is performed (5 minutes at $1200 \mathrm{rpm}$ speed).

After centrifugation, the erythrocytes were removed. The remaining material is centrifuged again (10 minutes at $1200 \mathrm{rpm}$ speed).

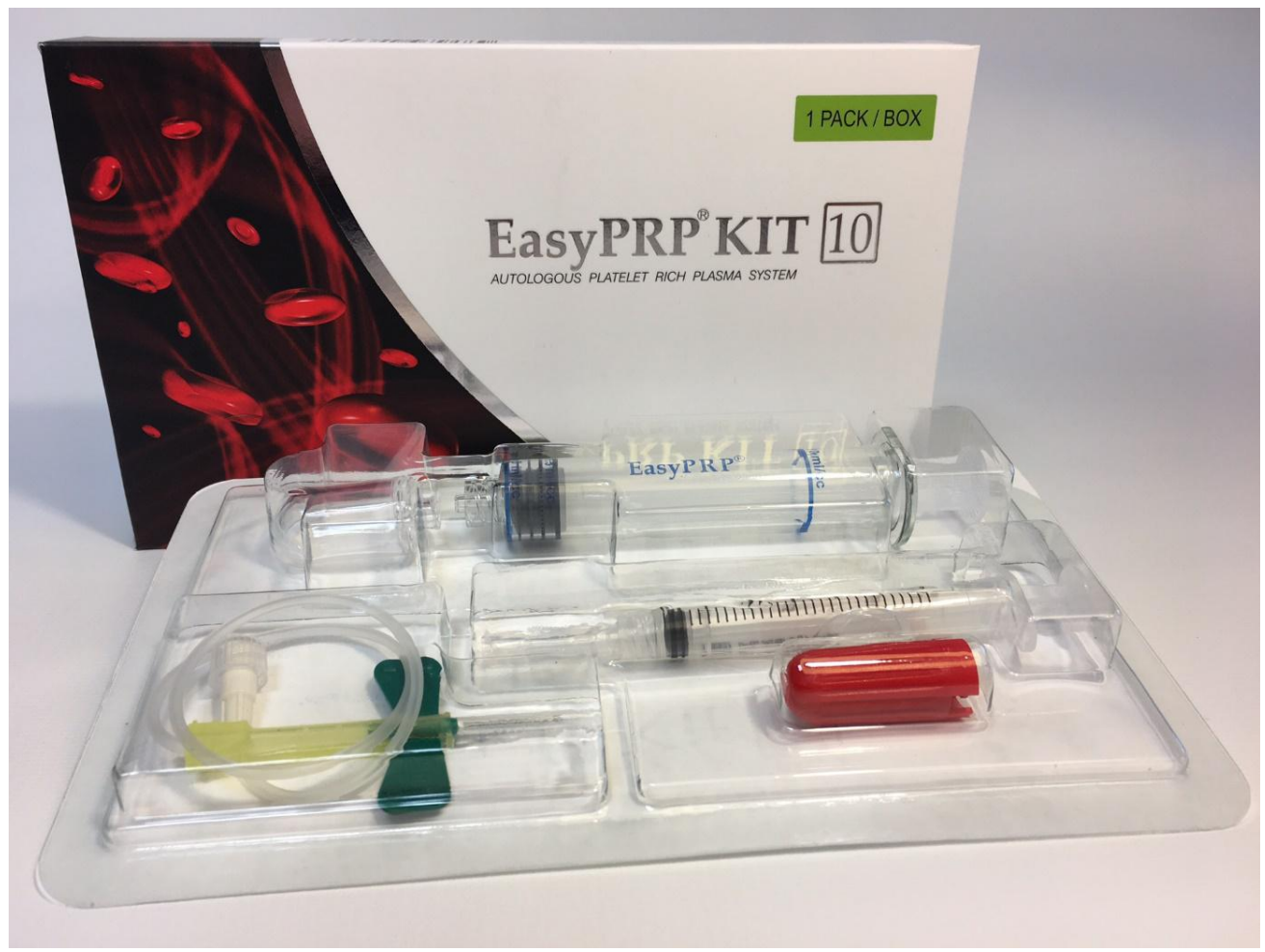

Image 2. Easy PRP kit (NEOTEC Bioteknoloji, Istanbul,Turkey)

At the bottom of the tube we can find three different layers with buffy coat (about $0.5 \mathrm{~mL}$ ), PPP (poor platelet plasma $2.5 \mathrm{~mL}$ ) as a second layer and PRP ( $3 \mathrm{~mL})$ as the third layer. Buffy coat and PPP are withdrawn with adjustable injector and PRP $(3 \mathrm{~mL})$ was injected into the knee joint using a lateral approach. [9, 12]

In the Third group: Ozone+PRP combination was injected to the knee of the patients weekly (PRP $+10 \mu \mathrm{g} / \mathrm{mL}$ with $5 \mathrm{~mL}$ ozone combination) in total 3 times.

We prepared the PRP as in the second group and took the ozone/ oxygen mixture from the medical ozone generator as in the first group. With a lateral approach to the knee, we first injected the PRP and the ozone gas immediately. 
Data were analyzed by statistical software package SPSS 17.0. Numerical variables were expressed as mean \pm standard deviation (SD). Paired sample t-test were used for comparison between pretreatment, post-treatment changes and intergroup variability. Statistical significance was set at $p<0.05$.

\section{Results}

According to the VAS, we got the following data (table 1):

Table 1. Paired samples T- test of VAS value.

\begin{tabular}{lccc}
$\begin{array}{l}\text { Treatment } \\
\text { options }\end{array}$ & $\begin{array}{c}\text { Pre-treatment } \\
\text { VAS }\end{array}$ & $\begin{array}{c}\text { Post-treatment } \\
\text { VAS }\end{array}$ & $\begin{array}{c}\% \text { improvement } \\
\text { VAS }\end{array}$ \\
\hline OZONE & $6.90+/-1.16$ & $2.41+/-1.7^{*}$ & $66.27+/-21.88$ \\
PRP & $7.21+/-0.9$ & $2.08+/-1.75^{*}$ & $71.78+/-23.27$ \\
OZONE+PRP & $7.15+/-1.19$ & $1.38+/-1.19^{*}$ & $81.13+/-17.16^{*}$ \\
\hline
\end{tabular}

*Statistical significance.

In the First Group: The VAS of 40 patients who had only Ozone injections decreased from 6.9 to 2.41 with statistical significance $(P<0.01)$.

In the Second Group: The VAS of 40 patients who received only PRP injections decreased from 7.21 to 2.08 with statistical significance $(P<0.01)$.

In the Third Group: The VAS of the 40 patients who received Ozone+PRP combination decreased from 7.15 to 1.38 with statistical significance $(P<0,01)$.

When the mean recovery ratio scores were evaluated, it was $66.27 \%$ in the ozone group, $71.78 \%$ in the PRP patients, and $81.13 \%$ in the Ozone+PRP combination.

As shown in table 1 , the recovery scores of the patients who received the Ozone+PRP combination were significantly better $(p<0.01)$ than ozone or PRP injections alone. The difference between groups 1 and 2 were not significant $(p=0.057)$.

No side effects were observed during or after the treatments.

\section{Discussion}

$\mathrm{OA}$ is a degenerative joint disease that increases with age and causes pain and disability to significantly quality of life.

It is considered to be an organ disease that produces cartilage changes in the subchondral bone, joints and tissues around the joints. Pathologically, destruction and loss of articular cartilage, subchondral sclerosis and osteophyte formation are often accompanied by synovial inflammation and destruction of other structures that support joint. The pain and a decrease in joint function impairs the patient to a wide variety of treatment seeking, but unfortunately there are no medical or 
physical methods that have scientifically proven to be effective in humans to prevent joint cartilage degradation. Therefore, all treatment approaches are focused on relieving pain and minimizing functional deficits.

The goal of treatment in knee OA should be to control pain, to preserve and correct joint functions, to provide functional independence and improve quality of life. In order to achieve these goals, knee OA therapy should include non-pharmacologic, pharmacologic and if necessary, surgical methods.

Treatment should be tailored specifically to each patient. Non-steroidal anti-inflammatory (NSAID) drugs are thought to be the standard treatment for $\mathrm{OA}$ in most cases but many patients are not able to tolerate these medications or are exposed to side effects; this is why only analgesics are adequate in some patients and in some we use hot and cold packs or some physical rehabilitation methods.

As we have seen in our study above; ozone or PRP injections alone seem to be useful for the patients with knee OA, but the results of Ozone+PRP injections in knee OA, reduced the pain significantly more than the Ozone or PRP injections alone.

This might be because of the anti-inflammatory effect of ozone has been supported by the release of anti-inflammatory growth factors from the platelets in PRP.

Comparing our data with the ones from the study of Khoshbin et al. [10], in our patients who received only intra articular PRP injections there was a VAS improvement from 7.21 to 2.08, but in Khoshbin's paper, they did not find any difference.

Comparing our data with the ones from the study of Lopes de Jesus et al. [11], in our patients who received only intra-articular ozone injections, we have got similar results in VAS improvement, although in our study we used $10 \mu \mathrm{g} / \mathrm{mL}$ and they used $20 \mu \mathrm{g} / \mathrm{mL}$ as ozone concentration. We have checked that using higher concentrations, the patients referred a burning sensation intra-articularly, so we use lower concentration of ozone to increase the comfort of the patient and get the similar result.

The patients who received both ozone and PRP have significantly better results in improving the VAS score than the patients receiving them separately. We have not found any similar paper in the scientific literature.

For future studies, we consider using ozone major autohemotherapy because of its anti-inflammatory and tissue restoring effect in addition to the injections of Ozone, PRP, Ozone+PRP [12].

\section{Conclusions}

This study suggests that using Ozone+PRP injections for patients who have knee pain with VAS over 5 due to OA grades 2-3 is the best option compared with Ozone or PRP injections alone. 


\section{References}

1. Kaçar C, Gilgil E, Urhan S, Arikan V, Dündar U, Oksüz MC, et al. The prevalence of symptomatic knee and distal interphalangeal joint osteoarthritis in the urban population of Antalya, Turkey. Rheumatol Int. 2005;25(3):201-204.

2. Bredveld FC. Osteoarthritis-the impact of a serious disease. Rheumatology (Oxford). 2004;43(Suppl.1):4-8.

3. Rilling S, Viebahn-Hansler R. The Use of Ozone in Medicine. New York: Haug; 1987.

4. Conaghan PG, Dickson J, Grant RL. Care and management of osteoarthritis in adults: summary of NICE guidance. BMJ. 2008;336(5): 502-503. doi: 10.1136/bmj.39490.608009.AD.

5. Dıraçoğlu D. Ozone-oxygen therapies in musculoskeletal diseases. Turk J Phys Med Rehab. 2016;62(2):183-191. doi: 10.5152/tftrd.2015.87262.

6. Manoto SL, Maepa MJ, Motaung SK. Medical ozone therapy as a potential treatment modality for regeneration of damaged articular cartilage in osteoarthritis. Saudi J Biol Sci. 2018 May;25(4):672-679. doi: 10.1016/j.sjbs.2016.02.002.

7. Laver L, Marom N, Dnyanesh L, Mei-Dan O, Espregueira-Mendes J, Gobbi A. PRP for degenerative cartilage disease: A systematic review of clinical studies. Cartilage. 2017 Oct;8(4):341-364. doi: 10.1177/1947603516670709.

8. Paoloni J, De Vos RJ, Hamilton B, Murrell GA, Orchard J. Platelet-rich plasma treatment for ligament and tendon injuries. Clin J Sport Med 2011;21:37-45. doi: 10.1097/JSM.0b013e31820758c7.

9. Chen Z, Deng Z, Ma Y, Liao J, Li Q, Li M, et al. Preparation procedures and evaluation of PRP injections in the treatment of knee osteoarthritis. J Vis Exp. 2019 Jan 4(143). doi: 10.3791/57700.

10. Khoshbin A, Leroux T, Wasserstein D, Marks P, Theodoropoulos J, Ogilvie-Harris $D$, et al. The efficacy of platelet-rich plasma in the treatment of symptomatic knee osteoarthritis: a systematic review with quantitative synthesis. Arthroscopy. 2013 Dec;29(12):2037-2048. doi: 10.1016/j.arthro.2013.09.006.

11. Lopes de Jesus CC, dos Santos FC, de Jesus LMOB, Monteiro I, Sant'Ana MSSC, Trevisani VFM. Comparison between intra- articular ozone and placebo in the treatment of knee osteoarthritis: a randomized, double-blinded, placebo-controlled study. PLOS ONE. 2017;12(7): e0179185. doi: 10.1371/journal.pone.0179185.

12. Bocci V. Autohaemotherapy after treatment of blood with ozone. A reappraisal. J Int Med Res. 1994;22:131-144. doi: $10.1177 / 030006059402200301$. 